\title{
Ocular surface complications result from dysregulation of the OGF-OGFr signaling pathway in female diabetic rats
}

\author{
INDIRA PURUSHOTHAMAN ${ }^{1}$, IAN S. ZAGON ${ }^{1}$, JOSEPH W. SASSANI ${ }^{2}$ and PATRICIA J. MCLAUGHLIN ${ }^{1}$ \\ Departments of ${ }^{1}$ Neural and Behavioral Sciences, and \\ ${ }^{2}$ Ophthalmology, Penn State University College of Medicine, Hershey, PA 17033, USA
}

Received January 26, 2021; Accepted March 30, 2021

DOI: $10.3892 /$ etm.2021.10119

\begin{abstract}
Approximately 4.5 million women in the United States exhibit diabetes-associated ocular complications. The time course and magnitude of these complications, and their association with the dysregulation of the opioid growth factor (OGF)-OGF receptor (OGFr) signaling pathway are unknown. The present study investigated the onset and magnitude of ocular surface complications and the association with a dysregulated OGF-OGFr signaling pathway in diabetic female rats. Adult female Sprague-Dawley rats were injected with streptozotocin in order to establish a model of type 1 diabetes (T1D), and a subset received insulin (T1D-INS). Blood glucose, body weight, tear production and corneal sensitivity, as well as serum and tissue expression levels of OGF and OGFr, were assessed. Corneal epithelial wound healing was also evaluated. In a second study, female T1D rats were treated with topical naltrexone (NTX) to determine whether blockade of the OGF-OGFr signaling pathway by NTX altered development of corneal surface complications. Female T1D rats had elevated glucose levels and reduced body weight compared with control and T1D-INS rats. In both diabetic groups, tear production was decreased within 2 weeks and corneal sensitivity was decreased 2.5-fold within 5 weeks, while corneal epithelial wound healing was delayed only in T1D rats. Serum and tissue levels of OGF and OGFr were elevated in diabetes. Twice daily NTX treatment reversed most ocular surface complications in the diabetic female rats. The present data demonstrated a seminal discovery in female T1D rats, in which the onset and magnitude of diabetes-associated ocular surface complications were associated with dysregulation of the OGF-OGFr regulatory pathway. Blockade of the
\end{abstract}

Correspondence to: Dr Patricia J. McLaughlin, Department of Neural and Behavioral Sciences, Penn State University College of Medicine, 500 University Drive, Hershey, PA 17033, USA

E-mail:pxm9@psu.edu

Abbreviations: NTX, naltrexone; OGF, opioid growth factor; OGFr, OGF receptor; STZ, streptozotocin; T1D, type 1 diabetes

Key words: T1D, NTX, dry eye, corneal surface sensitivity
OGF-OGFr pathway with the opioid receptor antagonist NTX prevented the onset and/or decreased the magnitude of these deficits. The current data support the need for translational research on this therapeutic approach for diabetic human subjects.

\section{Introduction}

Worldwide, diabetes is one of the fastest growing health challenges of the 21st century, with the number of adults diagnosed with diabetes tripling in the last two decades (1-3). By the year 2045 , it is estimated that $\sim 10 \%$ of the global population will be diagnosed with diabetes or pre-diabetes (1). In the United States, there are 27 million diabetic individuals, of which 11.7 million are females $(3,4)$. In addition to complications such as nephropathy and cardiovascular disease, diabetic individuals may also experience ocular complications $(5,6)$. In total, $>50 \%$ of individuals diagnosed with diabetes exhibit at least one ocular surface complication, such as dry eye disease, delayed corneal epithelial healing or keratopathy, during their lifetime, highlighting the unmet need for knowledge regarding the onset and course of diabetic ocular complications (5). Understanding the molecular mechanisms underlying the onset of these ocular defects may assist in developing therapeutics to manage them.

The Opioid Growth Factor (OGF)-OGF receptor (OGFr) pathway is active in corneal tissues, where it functions to maintain epithelial homeostasis of the cornea (7-9). Blockade of the OGF-OGFr pathway using naltrexone (NTX), an opioid antagonist, reverses numerous ocular epithelial complications in individuals with type 1 (T1D) or type 2 diabetes, including delayed epithelial wound healing, abnormal corneal sensitivity and low tear production (10-12). OGF, which is chemically termed $\left[\mathrm{Met}^{5}\right]$-enkephalin, is an endogenous pentapeptide that reduces cell replication, and its levels are elevated in diabetic humans (13-15) and animals (16). In addition, the effects of differences in the sex of the patient, the pathology of diabetes and the associated complications are reflected by the fact that female subjects are more likely to develop certain complications, whereas they are hormonally protected against others. Studies have shown the importance of sex hormones in ocular surface dysfunction, particularly in female subjects (17-19). At present, to the best of our knowledge, there are no studies using female animal models of T1D that document the timing and degree of dysregulation of the OGF-OGFr pathway 
in the cornea, or the effect of blockade of this pathway in preventing such abnormalities. In the present study, female Sprague-Dawley rats were treated with streptozotocin (STZ) to induce hyperglycemia to assess whether sex influenced the relationship between the tissue levels of OGF and OGFr in the corneal epithelium, and the onset and magnitude of corneal surface complications related to diabetes. The present study also investigated whether topical NTX treatment in female T1D rats could block the OGF-OGFr axis and prevent or delay diabetic complications of the ocular surface.

\section{Materials and methods}

Female T1D rat model. In the present study, 6-week old female Sprague Dawley rats weighing 110-120 g (Charles River, Laboratories, Inc.) were administered an intraperitoneal (i.p.) injection of $50 \mathrm{mg} / \mathrm{kg} \mathrm{STZ} \mathrm{(EMD} \mathrm{Millipore)} \mathrm{dissolved} \mathrm{in}$ sodium citrate buffer ( $\mathrm{pH} 4.5$ ), once per a day for 2 consecutive days. Rats were fasted overnight prior to injection of STZ. Blood glucose levels $>350 \mathrm{mg} / \mathrm{dl} 48-72 \mathrm{~h}$ after induction were indicative of successful establishment of the T1D rat model. A subset of T1D rats received insulin implants (T1D-INS; LinShin). The implants ( $7 \mathrm{~mm}$ ) were implanted subcutaneously on the left upper quadrant of the abdomen and were designed to release insulin for $45 \pm 2$ days at a rate of $2 \mathrm{U} / \mathrm{day}$. Control rats received i.p. injections of citrate buffer.

Blood glucose and body weight. Female rats were weighed and blood glucose levels were monitored on a weekly basis. The body weights, blood glucose levels, as well as non-invasive corneal surface parameters of 12 rats from each group were evaluated at each time point over the course of 8 weeks.

Evaluation of ocular surface complications. Corneal sensitivity and tear production measurements were assessed on unanesthetized rats, whereas corneal wounding was assessed under anesthesia. All procedures were performed under sterile conditions and in accordance with the ARVO Statement for the Use of Animals in Ophthalmic and Vision Research $(10,11)$.

Corneal sensitivity was evaluated by determining the blink rate using the Cochet-Bonnet aesthesiometer (Boca Raton) as described previously (11). The test was performed by obtaining three readings/eye/time point. The aesthesiometer pressure $\left(\mathrm{g} / \mathrm{mm}^{2}\right)$ was determined directly using the conversion table from the protocol supplied by the manufacturer.

Tear production was determined by inserting Schirmer strips (Alcon) into the lower lid, proximal to the lateral canthus for $1 \mathrm{~min}$, to evaluate the wetting length to the nearest half $\mathrm{mm}$, using a scale provided by the manufacturer. The Schirmer strips were cut into $1 \times 17 \mathrm{~mm}$ strips in a sterile environment and inserted into the cul-de-sac of unanesthetized rats, as described previously (11).

Corneal epithelial wound healing was determined following corneal abrasion (12). Surgeries were performed between 8 and 9 AM to prevent disparities in diurnal rhythm. Rats were anesthetized with a $0.1 \mathrm{ml}$ cocktail of ketamine $(100 \mathrm{mg} / \mathrm{kg})$, xylazine $(10 \mathrm{mg} / \mathrm{kg})$ and acepromazine (1 $\mathrm{mg} / \mathrm{kg}$ ) delivered by i.p. injection. A topical anesthetic, proparacaine-HCl (0.5\%; Bausch and Lomb), was applied to the cornea prior to creating a $5-\mathrm{mm}$ diameter abrasion in the right eye using a dermal trephine (Acuderm). The area of epithelium was abraded without penetrating below the level of the epithelium. The outlined epithelium was removed without damaging the underlying corneal tissue. Beginning at $16 \mathrm{~h}$, rats were anesthetized with $3 \%$ isoflurane (VEDCO) regulated with oxygen flow from a certified vaporizer, and the eyes were stained with fluorescein strips and imaged using cobalt blue filtered light to assess wound closure. Rats were not imaged prior to this $16 \mathrm{~h}$ period in order to ensure proper initiation of the epithelial wound healing process. Baseline images were taken at time $0,16,24,32,40$ and $48 \mathrm{~h}$ following wounding, and wound closure was documented as a percentage of the original wound area. After surgery, and as required, rats received buprenorphine $(0.025 \mathrm{mg} / \mathrm{kg}$; i.p.) every $8-12 \mathrm{~h}$ to offset any signs of pain (12).

Levels of OGF and OGFr in ocular tissue. Rats were humanely euthanized using an overdose of sodium pentobarbital ( $>150 \mathrm{mg} / \mathrm{kg})$. The eyes were removed from the orbital cavity, processed and immunohistochemically stained with OGF (Penn State University, 1:200) and OGFr (MyBioSource MBS2124207, 1:150) antibodies as described previously (16). Frozen corneal sections $(6-8 \mu \mathrm{m})$ from each group were stained following previously published protocols $(7,11,12,16)$; control sections were stained with the secondary antibody only (Alexa Flour568; Invitrogen; Thermo Fisher Scientific, Inc.). Stained sections were imaged using a Keyence Scope (KEYENCE, Ltd.) and further quantified using ImageJ 1.8.0_172 (National Institutes of Health) to quantify optical density (OD).

Serum levels of OGF, OGFr and the enkephalinase CD10 (also known as neprilysin) were measured in female rats using ELISA kits (MyBioSource) for CD10 (cat. no. MBS764927), OGF (cat. no. MBS756126) and OGFr (cat. no. MBS109224) (16). ELISA kits were specifically designed for rat serum, and experiments were performed according to the manufacturer's protocol. In order to ensure rigor and reproducibility, serum samples were utilized from multiple experiments and samples were replicated from other assays as additional positive controls. A minimum of three repeats of ELISA were performed with replicate samples from 6-8 different female rats at each time point.

Blockade of the OGF-OGFr pathway: Mechanistic approach to therapy. In order to evaluate the mechanisms underlying the interactions between ocular surface complications and the OGF-OGFr pathway, experiments were designed to block this pathway using NTX. Whether continuous opioid receptor blockade using topical NTX would prevent or substantially reduce the magnitude of ocular surface complications in female diabetic rats was assessed. A cohort of T1D female rats received one drop $(0.05 \mathrm{ml})$ of $5 \times 10^{-5}$ M NTX dissolved in saline (pH 7.0; T1D TopNTX-2Drop $_{\text {group) }}$ twice daily, as described previously $(11,12,16)$. Each drop was administered between 8 and 9 AM as well as between 3 and 4 PM for 4 weeks. For comparison, female rats in the T1D, T1D-INS and non-diabetic control groups not receiving NTX were included. The dose of NTX was based on previous studies which are reviewed elsewhere (8), and was considered an effective dosage to ensure prolonged blockade of the OGF-OGFr axis. 

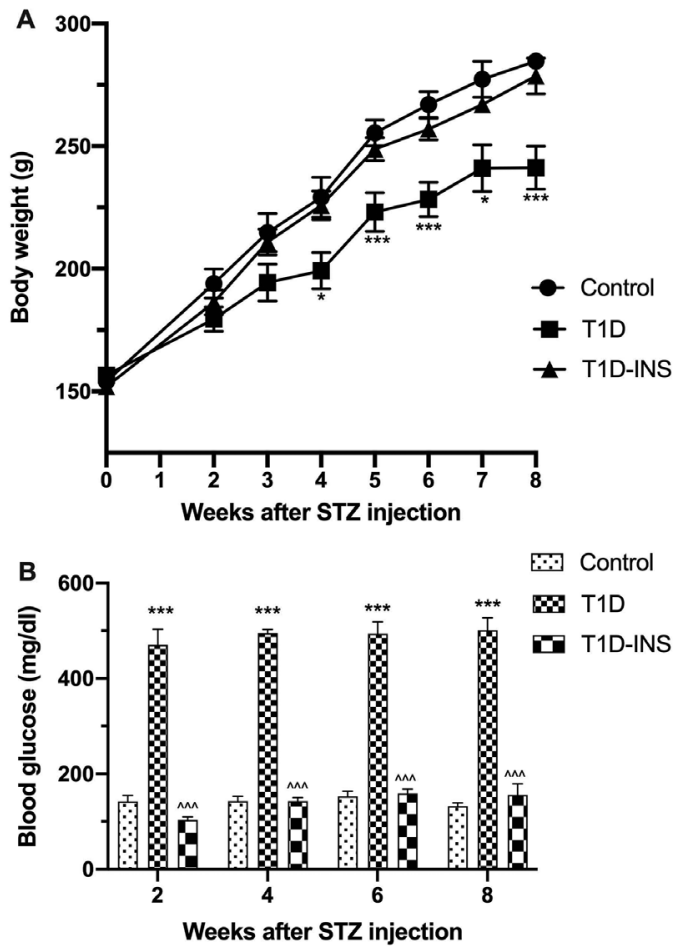

Figure 1. Body weights and blood glucose levels of female T1D, T1D-INS and control rats. (A) Body weights and (B) blood glucose levels for female Sprague-Dawley rats randomly selected from T1D, T1D-INS or control groups ( $\mathrm{n} \geq 11$ per group). The data are presented as the mean $\pm \mathrm{SE}$. ${ }^{*} \mathrm{P}<0.05$ and ${ }^{* * *} \mathrm{P}<0.001$ vs. control; ${ }^{\wedge \wedge} \mathrm{P}<0.001$ vs. T1D. T1D, type 1 diabetes; INS, insulin; STZ, streptozotocin.

Statistical analysis. A total of five independent experiments were performed. Multiple experiments were performed with a smaller number of rats per group in order to ensure rigor and reproducibility. For all parameters assessed, 12-15 rats per treatment condition were evaluated. Data were analyzed using a one- or two-way ANOVA. If interactions were significant, a Tukey post-hoc test was used for multiple comparisons. Analysis was performed using GraphPad Prism version 8.0 (GraphPad Software, Inc.). P $<0.05$ was considered to indicate a statistically significant difference.

\section{Results}

Female TID rat model phenotype. Female STZ-treated rats presented with a blood glucose level $>300 \mathrm{mg} / \mathrm{dl}$ within $48-72 \mathrm{~h}$ post-STZ injection. Insulin implants were introduced into 13 rats. Of the 64 rats injected with STZ, five rats did not become hyperglycemic and four rats died within the first 2 weeks of STZ exposure. Fig. 1A and B present the body weights and blood glucose levels of the rats, respectively, showing that female T1D rats gained less weight over the 8 -week period relative to the control and T1D-INS female rats. The body weights of the control female rats increased by a mean of $154 \mathrm{~g}$ from baseline to $284 \mathrm{~g}$ by week 8 , whereas the T1D rats only exhibited an increase to $241 \mathrm{~g}$ by week 8 . T1D-INS rats weighed $\sim 278 \mathrm{~g}$ by week 8 . Blood glucose levels of the control rats ranged between $130-150 \mathrm{mg} / \mathrm{dl}$, whereas glucose levels were measured in the T1D between $456-470 \mathrm{mg} / \mathrm{dl}$. Glucose levels in the T1D-INS rats ranged between $103-155 \mathrm{mg} / \mathrm{dl}$.

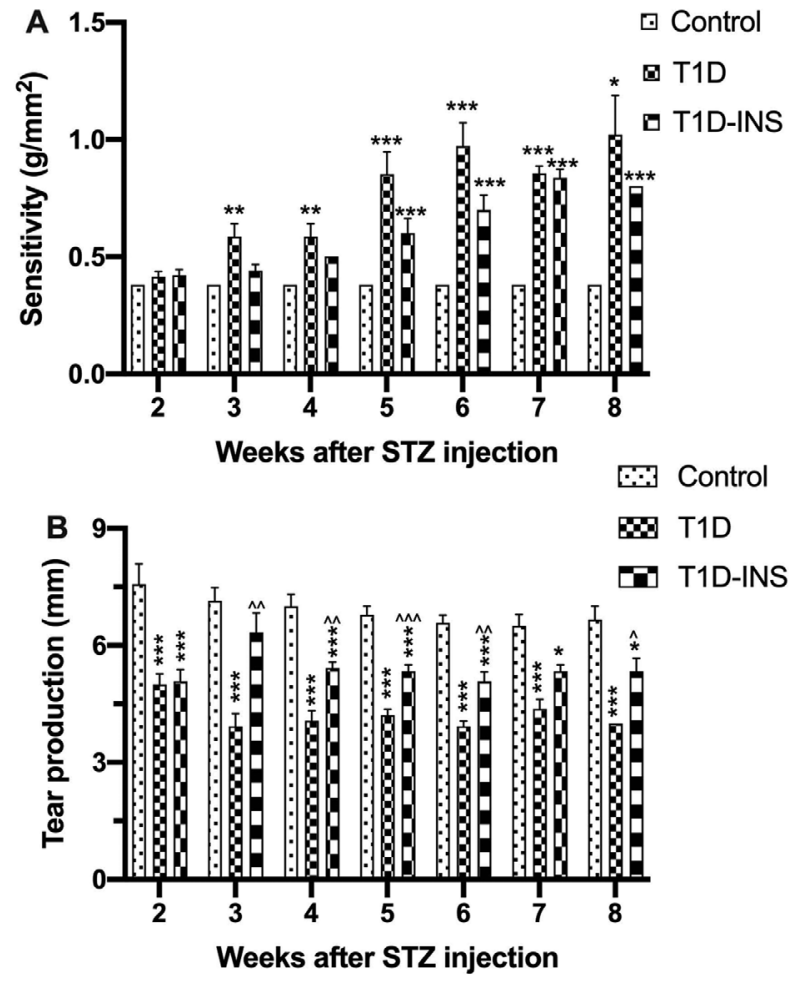

Figure 2. Corneal surface sensitivity and tear production of female T1D, T1D-INS and control rats. (A) Corneal surface sensitivity was measured with an aesthesiometer. Four readings of pressure were averaged for each rat at each time point and converted to sensitivity using the manufacturer provided conversion table (Cochet-Bonnet; Boca Raton). (B) Tear production was measured by the Schirmer tear test. The data are presented as the mean \pm SE for female rates randomly chosen at each time point from all groups $(\mathrm{n} \geq 11$ per group). ${ }^{*} \mathrm{P}<0.05,{ }^{* *} \mathrm{P}<0.01$ and ${ }^{* * *} \mathrm{P}<0.001$ vs. control; ${ }^{\wedge} \mathrm{P}<0.05,{ }^{\wedge} \mathrm{P}<0.01$ and ${ }^{\wedge \wedge} \mathrm{P}<0.001$ vs. T1D. T1D, type 1 diabetes; INS, insulin; STZ, streptozotocin.

Ocular surface defects in the TID female rats. Beginning at week 2 post-STZ injection, corneal surface sensitivity (Fig. 2A) and tear production (Fig. 2B) were evaluated weekly. On week 4, the results showed that the onset of significant changes in esthesiometry in T1D rats significantly decreased compared with the control group. Both the uncontrolled and controlled diabetic groups had reduced corneal surface sensitivity on weeks $5-8$, requiring greater pressure to induce a positive blink response. Female T1D rats required $\sim 0.58 \mathrm{~g} / \mathrm{mm}^{2}$ pressure on week 4 and $1.02 \mathrm{~g} / \mathrm{mm}^{2}$ pressure by week 8 to elicit a blink response. The pressure required for the T1D-INS rats was $\sim 0.50 \mathrm{~g} / \mathrm{mm}^{2}$ at week 4 , but this increased to $0.8 \mathrm{~g} / \mathrm{mm}^{2}$ by week 8 , whereas the control rats required a constant pressure of $0.38 \mathrm{~g} / \mathrm{mm}^{2}$ throughout the entirety of the study (Fig. 2A). Tear production was used to assess dry eye conditions, and was measured as the length of wetting on the Schirmer strips. Tear production for the control rats ranged between 6.5-7.6 $\mathrm{mm}$ wetting distance, whereas both T1D and T1D-INS had decreased tear production 2 weeks after STZ exposure. T1D rats exhibited a steady decline in tear production from week 2 starting at an average of $5 \mathrm{~mm}$ and dropping to $4 \mathrm{~mm}$ on week 8 , whereas the T1D-INS rats exhibited wetting distances between $5.5-6.5 \mathrm{~mm}$ over the 8 weeks.

Rate of corneal surface healing in the female TID model. Corneal surface wound healing was assessed at week 6 


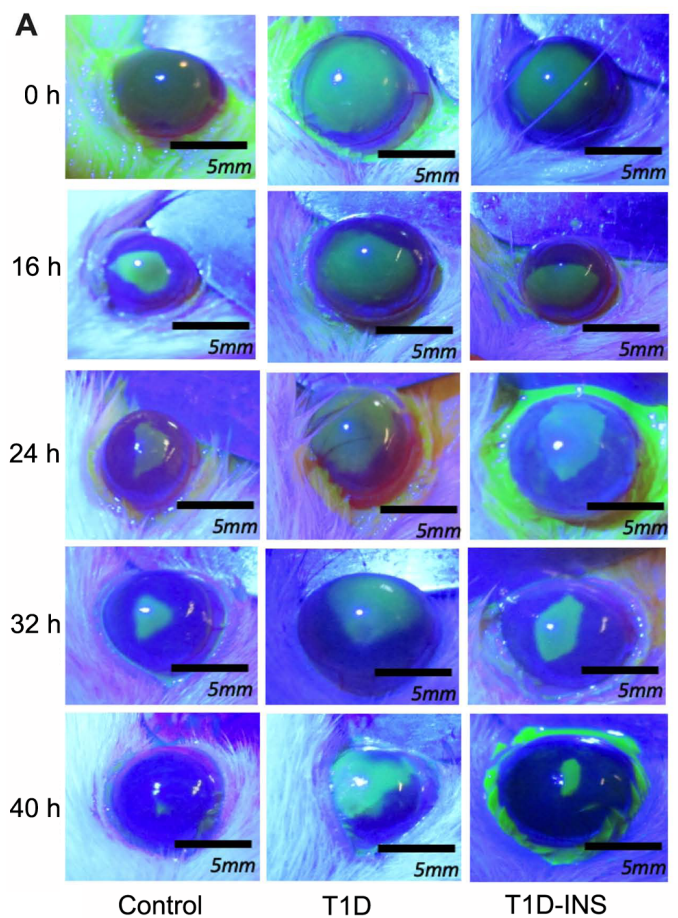

B

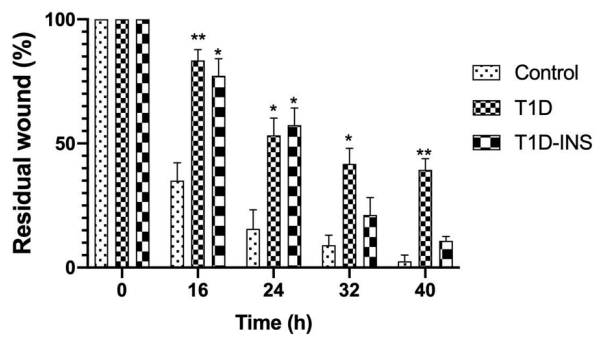

D

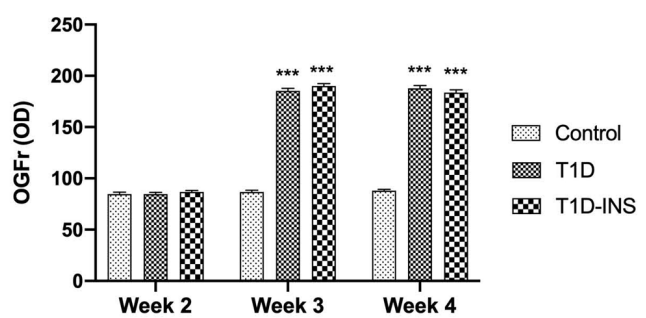

Figure 3. Photographs and percent residual corneal defects in T1D, T1D-INS and control rats. (A) Photographs (scale bar, 5 mm) and (B) percent residual corneal defects in T1D, T1D-INS and control rats. Eyes were stained with fluorescein and photographed prior to wounding ( 0 h) and at $16,24,32$ and $40 \mathrm{~h}$ after abrasion. Images with residual defects underwent areal analyses and are presented as the percentage of the original wound for the same rat until healed. (C) Expression patterns of OGF and OGFr. Immunohistochemically stained sections of corneal epithelium at 2,3 and 4 weeks following hyperglycemia in control, T1D and T1D-INS rats. Images were scanned, and (C) OGF and (D) OGFr expression was determined using ImageJ calibrated to measure OD. Values represent the mean $\pm \mathrm{SE}$ for 20 readings from 3 slides from each of 3 tissue specimens. ${ }^{*} \mathrm{P}<0.05,{ }^{* *} \mathrm{P}<0.01$ and ${ }^{* * *} \mathrm{P}<0.001$ vs. control. OD, optical density; T1D, type 1 diabetes; INS, insulin; OGF, opioid growth factor; OGFr, OGF receptor.

following the creation of a 5-mm central corneal epithelial wound (Fig. 3A). At $16 \mathrm{~h}$ post wounding, the T1D rats exhibited significantly delayed wound healing compared with the control rats. The T1D rats had $80 \%$ of their original wound still present, whereas the control rats had only $34 \%$ of their original wound area present. After $40 \mathrm{~h}$, the wounds in the control rats were completely healed ( $<1 \%$ residual wound), whereas in the T1D group, $40 \%$ of the wound area remained, and in the T1D-INS group, 10\% remained (Fig. 3B). Wound closure did not differ between the T1D-INS rats and the control group, suggesting that systemic administration of insulin may improve re-epithelialization of the cornea.

Ocular surface tissue and serum OGF and OGFr expression levels. The expression levels of OGF and OGFr in corneal epithelial tissues were semi-quantitatively measured after 2, 3 and 4 weeks (Fig 3C and D). OGF expression was $~ 88$ OD units in all groups at week 2. OGF expression levels were significantly elevated in both diabetic groups at week 3 and 4;
239 OD units in the T1D rats and 203 OD units in the T1D-INS rats. Similarly, OGFr expression levels in the corneal epithelium were comparable between all groups at week 2 . OGFr expression at week 3 post-STZ in the control rats was $\sim 86$ OD units, and was elevated in both diabetic groups. Expression of OGFr in corneal epithelial tissue increased to $>95$ OD units by week 8 in both T1D and T1D-INS groups. Insulin treatment did not reduce the OGF or OGFr levels in the diabetic rats.

Serum OGF levels of the control, T1D and T1D-INS groups were comparable to each other 2 weeks after STZ injection (Fig. 4A). At week 3, T1D and T1D-INS OGF levels increased, and by week 4 they were 3 -fold greater than the OGF values at baseline or in the non-diabetic group. Serum OGF levels in the T1D rats remained elevated throughout the study. T1D-INS rats had elevated OGF serum levels compared with the control group, with significantly higher values at weeks 4 and 8 . Relative to OGF values, OGFr serum levels were $\sim 1,000$-fold higher in female rats (Fig. 4B). Mean OGFr levels for the control female rats ranged from 2.4-3.5 ng/ml over an 8-week 

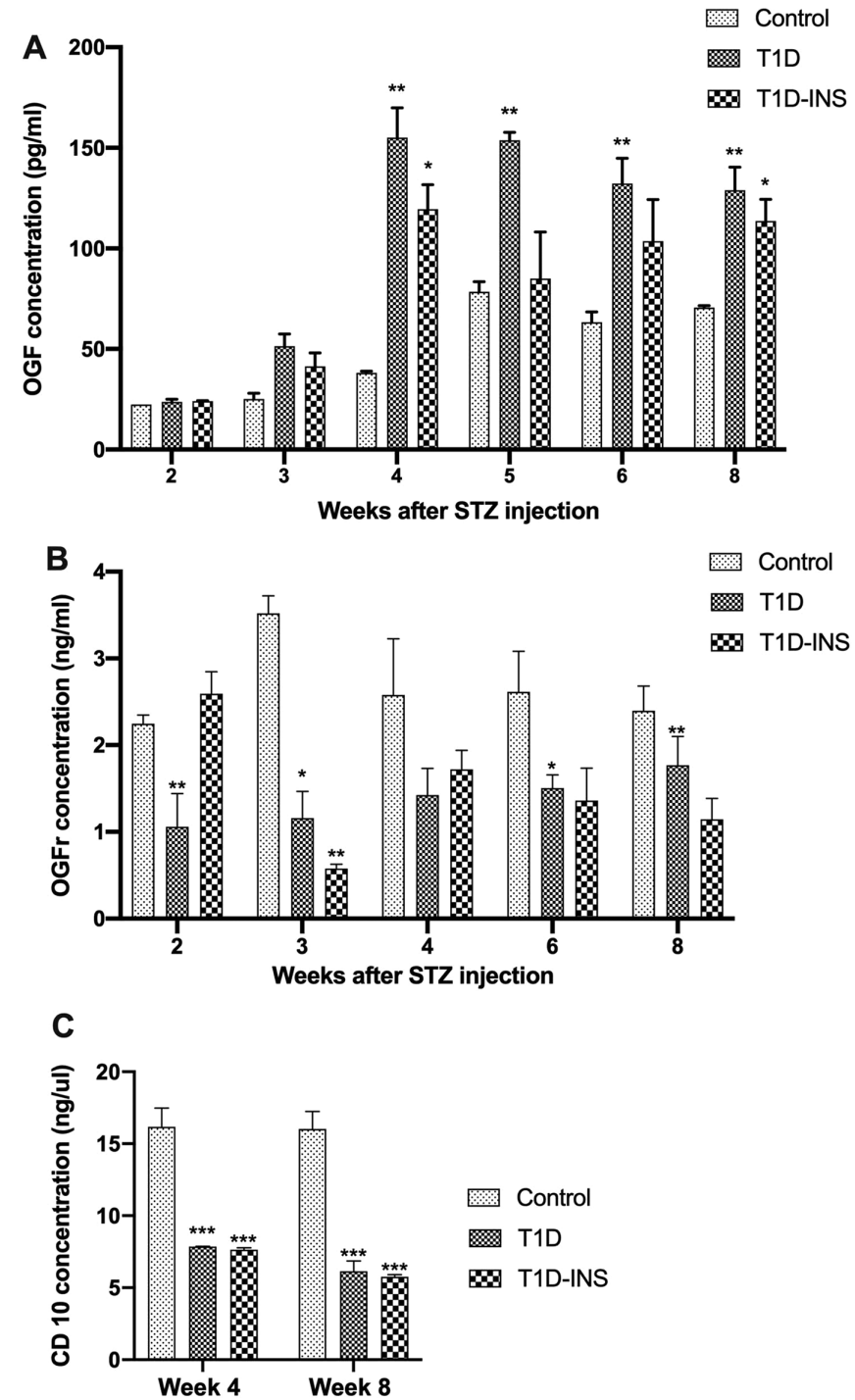

Figure 4. Serum levels of female T1D, T1D-INS and control rats. Serum levels of (A) OGF, (B) OGFr and (C) CD10 were measured weekly following hyperglycemia in control, T1D and T1D-INS female rats. The data are presented as the mean \pm SE for values from 4 different ELISA tests, and were analyzed using one-way ANOVA and Tukey's post-hoc test. ${ }^{*} \mathrm{P}<0.05,{ }^{* * *} \mathrm{P}<0.01$ and ${ }^{* * * *} \mathrm{P}<0.001$ vs. control. T1D, type 1 diabetes; INS, insulin; STZ, streptozotocin; OGF, opioid growth factor; OGFr, OGF receptor.

period. A two-way ANOVA did not reveal a significant difference between time point and treatment. OGFr values were lower in the T1D group compared with the control group at all time points, and this decrease was significant at weeks 2 , 3,4 and 8 . T1D-INS rats had comparable levels to the control rats, except at week 4 when there was a significant decrease in OGFr levels detected in the serum of the T1D-INS rats. CD10 is an enkephalinase that metabolizes OGF. At weeks 4 and 8 post-STZ injection, controlled and uncontrolled diabetic rats had a significantly reduced CD10 concentration relative to the control level of $\sim 16 \mathrm{ng} / \mu \mathrm{l}$, with the levels in the diabetic rats being 2 -fold less (Fig. 4C).

Treatment of ocular surface complications by blocking the OGF-OGFr pathway. The second part of this study focused on the impact of topical NTX treatment to block the interaction between OGF and OGFr, and to determine whether dysregulation of this pathway led to ocular surface defects. At 2, 3 and 4 weeks post-STZ injection, corneal surface sensitivity (Fig. 5A) and tear production (Fig. 5B) were evaluated. All groups recorded a required pressure of $0.38 \mathrm{~g} / \mathrm{mm}^{2}$ at week 2 for eliciting a blink response. T1D rats demonstrated significantly decreased corneal sensitivity on weeks 3 and 4 relative to the control, requiring twice the pressure to elicit a positive blink response. T1D-INS rats had comparable sensitivity to the control rats in the first 4 weeks post-STZ. However, $\mathrm{T}^{\mathrm{D}} \mathrm{D}_{\mathrm{TopNTX} \text {-2Drop }}$ rats were comparable to the control rats throughout the entirety of the study. Measurement of tear volume showed the rats in the control group had Schirmer strip wetting measurements of 7-8 mm over the course of 4 weeks. Female T1D and T1D-INS rats exhibited reduced tear production beginning on week 2, and it ranged between 4-5 $\mathrm{mm}$ in week 4 post-STZ. However, topical administration of NTX restored tear production, such that rats in the $\mathrm{T}_{1} \mathrm{D}_{\text {TopNTX-2Drop }}$ group had tear volume production levels comparable to the control rats at all time points, ranging from $6.8-8.0 \mathrm{~mm}$. $\mathrm{T}_{1} \mathrm{D}_{\text {TopNTX-2Drop }}$ values were significantly higher than that in the T1D rats at weeks 2, 3 and 4, and from T1D-INS rats at week 4. Serum levels of OGF (Fig. 5C) and OGFr (Fig. 5D) were monitored only at week 4 . The results showed that all diabetic groups exhibited significantly increased serum levels of OGF relative to the levels observed in the control rats $(\sim 47 \mathrm{pg} / \mathrm{ml})$.

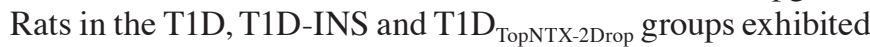
a $>2$-fold increase in the serum levels of OGF, with values of $\sim 154, \sim 125$ and $137 \mathrm{pg} / \mathrm{ml}$, respectively. OGFr serum concentrations did not differ amongst groups (Fig. 5D).

OGF (Fig. 5E and F) and OGFr (Fig. 5G and H) expression levels in the corneal epithelial tissue were also assessed on week 4 in the corneal epithelium treated topically with NTX. Compared with the expression levels of the control rats, the levels of OGF in the T1D and T1D-INS rat tissues were significantly increased, with levels of 219 and 202 OD units, respectively, whereas the $\mathrm{T}_{1} \mathrm{D}_{\text {TopNTX-2Drop }}$ NTX-treated rats had OGF levels comparable to that of the control rats ( 87 OD units). The value of the $\mathrm{T}_{1} \mathrm{D}_{\mathrm{TopNTX}-2 \mathrm{Drop}}$ group was $92 \mathrm{OD}$ units, and this was significantly lower compared with the T1D rats. Similarly, OGFr expression levels were elevated in the corneal epithelium of T1D and T1D-INS rats (180 and 192 OD units, respectively) compared with the control rats (80 OD units). $\mathrm{T}_{1} \mathrm{D}_{\text {TopNTX-2Drop }}$ treated rats had a substantially reduced OGFr value of 88 OD units, similar to that of the control rats, and significantly lower than that of the T1D rats.

\section{Discussion}

In the present study, an association between ocular surface abnormalities and the dysregulation of the OGF-OGFr pathway in female diabetic rats was demonstrated. In STZ-induced hyperglycemic female rats, systemic administration of insulin prevented elevations in blood glucose levels and weight loss associated with hyperglycemia over an 8 -week period. Systemic insulin treatment in female diabetic rats resulted in Control rates of corneal epithelial wound healing, but did not protect against other ocular surface complications. The present study is the first to report the relative expression of OGF and OGFr in the corneal epithelium over the course of 8 weeks, in uncontrolled and insulin controlled diabetic female rats. Systemic insulin 
A

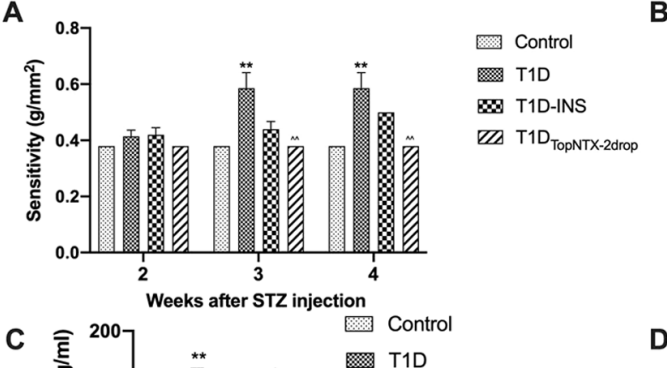

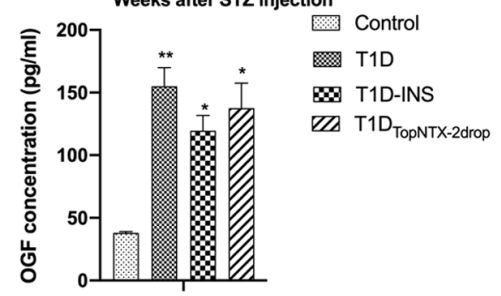
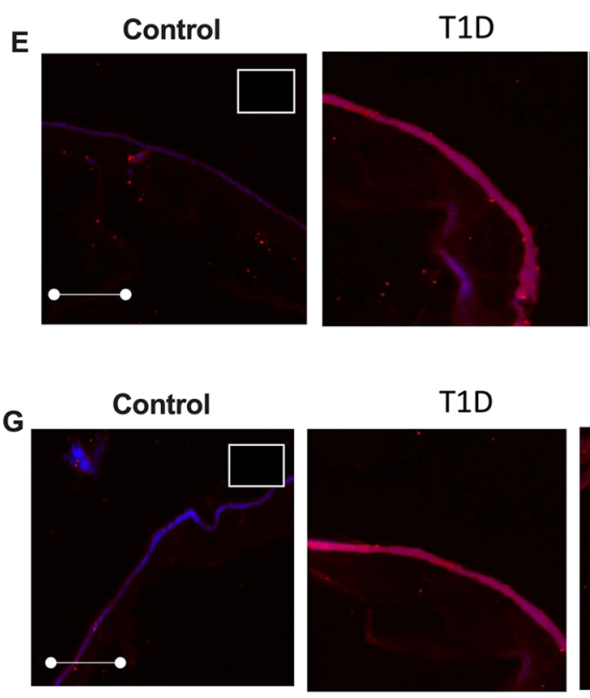
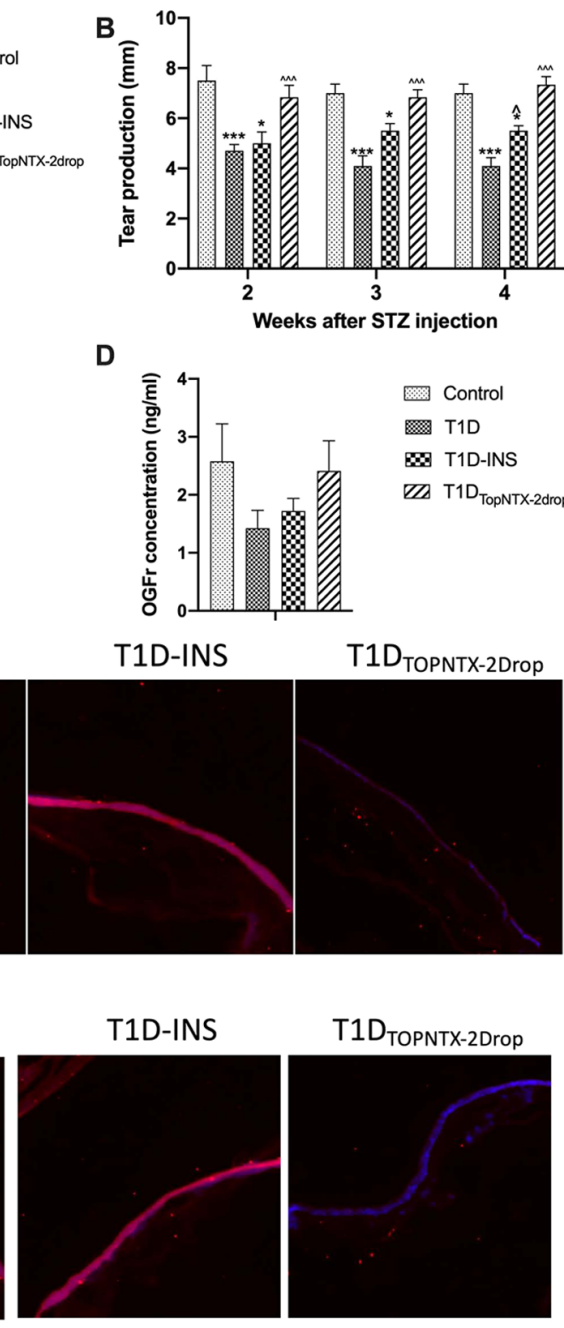

D

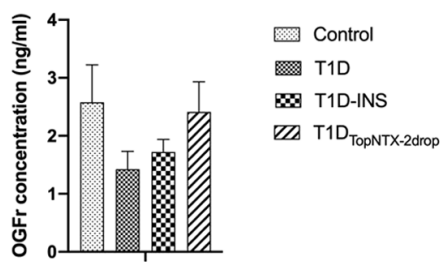

T1D-INS
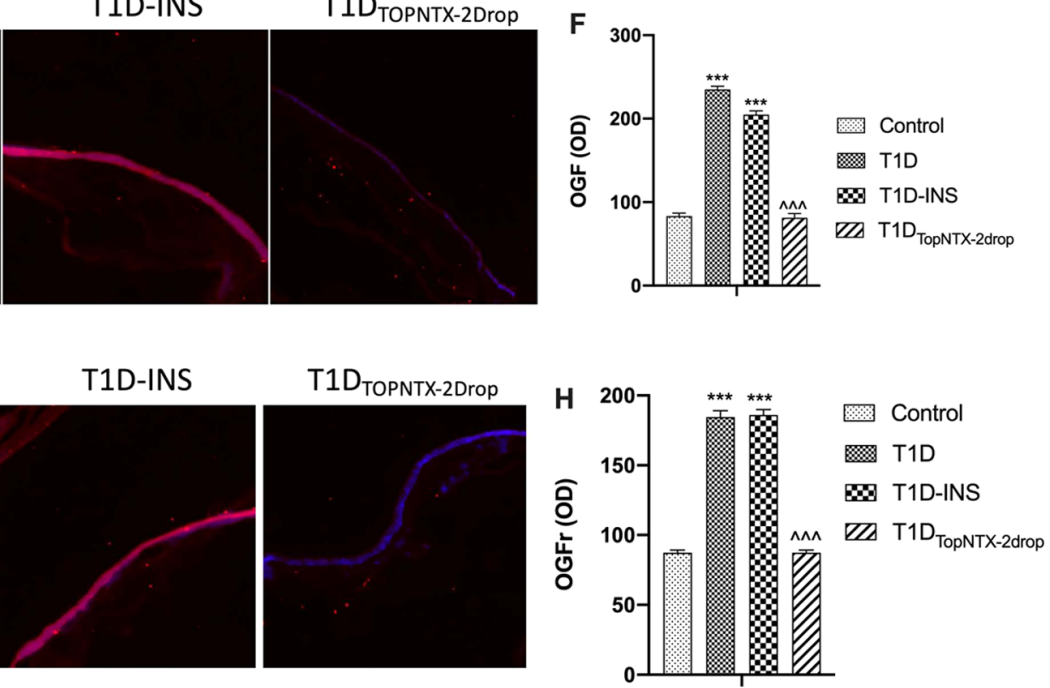

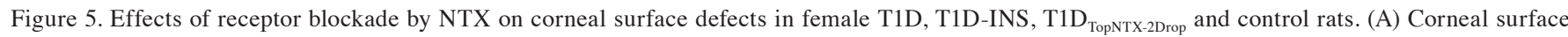
sensitivity was measured with an aesthesiometer and 4 readings of pressure were averaged at each time point for each rat and converted to sensitivity using the conversion table provided by the manufacturer (Cochet-Bonnet; Boca Raton). (B) Tear production was measured by the Schirmer tear test. Values are presented as the mean \pm SE for female rates randomly chosen at each time point from all groups ( $\geq 13$ per group). Serum levels of (C) OGF and (D) OGFr were measured

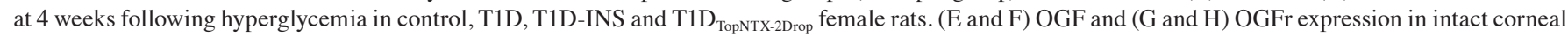

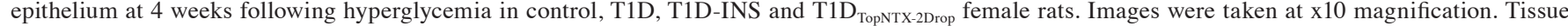
stained with only secondary antibody is shown in the white outlined insert located in control images in the upper right corner. Quantitative evaluation of OGF and OGFr expression was based on scanned confocal images and evaluated using ImageJ software. Values are presented as the mean \pm SE for $18-20$ readings collected from $\geq 4$ tissue specimens. ${ }^{*} \mathrm{P}<0.05,{ }^{* *} \mathrm{P}<0.01$ and ${ }^{* * * *} \mathrm{P}<0.001$ vs. control; ${ }^{\wedge} \mathrm{P}<0.05,{ }^{\wedge} \mathrm{P}<0.01$ and ${ }^{\wedge \wedge} \mathrm{P}<0.001$ vs. T1D. T1D, type 1 diabetes; INS, insulin; STZ, streptozotocin; OGF, opioid growth factor; OGFr, OGF receptor; NTX, naltrexone; OD, optical density.

treatment did not prevent the increase in OGF or OGFr expression in corneal epithelium, nor did it reduce the magnitude of ocular complications. The efficacy of OGF-OGFr blockade with NTX was assessed in female rats. NTX treatment was capable of restoring tear production and corneal surface sensitivity, and appeared to be more effective than insulin treatment in addressing the ocular surface abnormalities.

In a previous study using the diabetic male rat model, tear production and corneal sensitivity were altered 4 weeks after STZ injection, with a corresponding increase in the serum levels of OGF (16), whereas the onset of these ocular surface complications occurred earlier in the diabetic female animals. Female T1D and T1D-INS rats exhibited significant abnormalities with regard to corneal sensitivity and tear production 2 weeks after STZ injections relative to the male rats after 4 weeks (16). In the present study, female T1D rats healed in $\sim 50 \%$ of the time it took for the male T1D rats to heal.
The interaction between insulin and the OGF-OGFr pathway is unknown. The findings in the present study, as well as data on male rats (16), suggest that there are potentially two pathways modulating corneal surface complications in diabetes. One pathway is related to the delayed epithelial wound healing, which appears to benefit from systemic administration of insulin, as female rats in the T1D-INS group exhibited relatively normal rates of repair. In the present and previous studies $(20,21)$, insulin provided a level of protection against delayed re-epithelialization. However, control of hyperglycemia through insulin did not affect the decrease in tear production or altered corneal sensitivity. Moreover, OGF and OGFr levels were dysregulated in both controlled and uncontrolled T1D, suggesting that a separate upstream pathway caused dysregulation of the OGF-OGFr axis, which then subsequently altered peptide and receptor levels.

The elevated levels of OGF and OGFr in epithelial tissue occurred earlier in diabetic female animals compared with 
the male T1D animals, and the severity of the ocular defects were increased 3-fold compared with the magnitude recorded in male T1D animals (16). There were differences in the serum OGF and OGFr levels based on sex; baseline serum OGF levels in female animals were twice that of males (16), and hyperglycemia resulted in an increase in OGF levels in female T1D animals that were almost 3-fold greater than the increase observed in the male T1D rats, and $>6$-fold higher than that of the baseline. These data suggest that sex serves a role in the onset and magnitude of diabetic ocular complications, and this observation is supported by previous studies $(16,19,21,22)$. Thus, additional investigations into the role that male and female hormones serve in the regulation of the OGF-OGFr pathway are required.

Information on the OGF-OGFr pathway in female rats is limited, and the impact of dysregulation of the OGF-OGFr pathway in female T1D rats remains to be assessed. Blockade of the OGF-OGFr pathway using the opioid receptor antagonist NTX, reversed ocular surface abnormalities in a male diabetic animal model $(10-12,16)$, leading to the hypothesis that corneal surface abnormalities and the dysregulation of the OGF-OGFr pathway may also be correlated with a female diabetic rat model. However, there are sex differences that result in varied responses between male and female mice. To the best of our knowledge, the present study was the first to show an association between the onset of ocular surface abnormalities and elevated OGF serum levels in female diabetic rats. Furthermore, it was the first study to investigate continuous blockade of OGFr using topical administration of NTX as a treatment for ocular surface complications in female T1D rats. These findings extend and corroborate those of a previous study, which used a male diabetic animal model (16), and suggest that both male and female rats with hyperglycemia are vulnerable to the effects of a dysregulated OGF-OGFr pathway. Moreover, topically administered NTX is a safe and effective treatment modality $(8,23)$, and based on the results of the present study, may also be an effective means of preventing and/or treating ocular surface complications associated with diabetes in female rats.

In conclusion, the results of the present study support the hypothesis that dysregulation of the OGF-OGFr pathway in female T1D rats is associated with the onset of ocular surface complications, and that blockade of this pathway via topical administration of NTX prevents and/or reduces the magnitude of dry eye and corneal surface sensitivity. These results may assist in the discovery of specialized treatment options for diabetic female patients, who are disproportionately affected by corneal surface complications.

\section{Acknowledgements}

The authors would like to thank Dr Chirag L. Patel from Penn State College of Medicine (Hershey, USA) for his support during this study.

\section{Funding}

The present study was supported by a grant from the National Institutes of Health [grant no. EY029223 (ISZ)].

\section{Availability of data and materials}

The datasets used and/or analyzed during the current study are available from the corresponding author on reasonable request.

\section{Authors' contributions}

ISZ, PJM and JWS conceived the study. IP performed the experiments, and acquired and analyzed the data. PJM and ISZ confirm the authenticity of all the raw data. IP, JWS, ISZ and PJM interpreted the data, and wrote and revised the manuscript. All authors read and approved the final manuscript.

\section{Ethics approval and consent to participate}

The present study (protocol 47207) was approved by the Penn State College of Medicine Institutional Animal Care and Use Committee (Hershey, USA).

\section{Patient consent for publication}

Not applicable.

\section{Competing interests}

PJM, ISZ and JSW have intellectual property owned by Penn State Research Foundation that involves naltrexone treatment of the ocular surface, but receive no financial compensation or royalties. IP declares to have no competing interests.

\section{References}

1. Cho NH, Shaw JE, Karuranga S, Huang Y, da Rocha Fernandes JD, Ohlrogge AW and Malanda B: IDF diabetes atlas: Global estimates of diabetes prevalence for 2017 and projections for 2045. Diabetes Res Clin Pract 138: 271-281, 2018.

2. International Diabetes Federation. Worldwide toll of diabetes. IDF Diabetes Atlas, 9th edition. 2019. https://www.diabetesatlas. org/en/. Accessed December 2020.

3. Centers for Disease Control and Prevention. National Diabetes Statistics Report, 2020. Atlanta, GA: Centers for Disease Control and Prevention, U.S. Department of Health and Human Services. Available from: https://www.cdc.gov/diabetes/pdfs/data/statistics/national-diabetes-statistics-report.pdf Accessed January 2021.

4. Moeineslam M, Amiri P, Karimi M, Jalali-Farahani S, Shiva N and Azizi F: Diabetes in women and health-related quality of life in the whole family: A structural equation modeling. Health Qual Life Outcomes 17: 178, 2019.

5. American Diabetes Association. Complications. https://www. diabetes.org/diabetes/complications Accessed December 2020.

6. Vieira-Potter VJ, Karamichos D and Lee DJ: Ocular complications of diabetes and therapeutic approaches. Biomed Res Int 2016: 3801570, 2016

7. Zagon IS, Sassani JW and McLaughlin PJ: Reepithelialization of the human cornea is regulated by endogenous opioids. Invest Ophthalmol Vis Sci 41: 73-81, 2000.

8. Sassani JW, McLaughlin PJ and Zagon IS: The yin and yang of the opioid growth regulatory system: Focus on diabetes-the Lorenz E. Zimmerman tribute lecture. J Diabetes Res 2016: 9703729, 2016.

9. Zagon IS, Sassani JW, Verderame MF and McLaughlin PJ: Particle-mediated gene transfer of OGFr cDNA regulates cell proliferation of the corneal epithelium. Cornea 24: 614-619, 2005.

10. Zagon IS, Sassani JW, Immonen JA and McLaughlin PJ: Ocular surface abnormalities related to type 2 diabetes are reversed by the opioid antagonist naltrexone. Clin Exp Ophthalmol 42: 159-168, 2014.

11. Zagon IS, Klocek MS, Sassani JW and McLaughlin PJ: Dry eye reversal and corneal sensation restoration with topical naltrexone in diabetes mellitus. Arch Ophthalmol 127: 1468-1473, 2009. 
12. Klocek MS, Sassani JW, McLaughlin PJ and Zagon IS: Topically applied naltrexone restores corneal reepithelialization in diabetic rats. J Ocul Pharmacol Ther 23: 89-102, 2007.

13. Negri M, Fallucca F, Tonnarini G, Mariani P, D'alessandro M and Pachí A: High levels of circulating met-enkephalin in pregnant and menstruating type 1 diabetic women. Gynecol Endocrinol 4: 25-31, 1990.

14. Fallucca F, Tonnarini G, Di Biase N, D'Alessandro M and Negri M: Plasma met-enkephalin levels in diabetic patients: Influence of autonomic neuropathy. Metabolism 45: 1065-1068, 1996.

15. Negri M, Tonnarini G, D'Alessandro M and Fallucca F: Plasma met-enkephalin in type I diabetes. Metabolism 41: 460-461, 1992.

16. Zagon IS, Sassani JW, Purushothaman I and McLaughlin PJ: Dysregulation of the OGF-OGFr pathway correlates with elevated serum OGF and ocular surface complications in the diabetic rat. Exp Biol Med (Maywood) 245: 1414-1421, 2020.

17. Vistisen D, Witte DR, Tabák AG, Brunner EJ, Kivimäki M and Færch K: Sex differences in glucose and insulin trajectories prior to diabetes diagnosis: The Whitehall II study. Acta Diabetol 51: 315-319, 2014.

18. Bowden MA, Tesch GH, Julius TL, Rosli S, Love JE and Ritchie RH: Earlier onset of diabesity-induced adverse cardiac remodeling in female compared to male mice. Obesity (Silver Spring) 23: 1166-1177, 2015.
19. Gagliano C, Caruso S, Napolitao G, Malaguarnera G, Cicinelli NV, Amato R, Reibaldi M, Incarbone G, Bucolo C, Drago F and Avitabile T: Low levels of 17- $\beta$-oestradiol, oestrone and testosterone correlate with severe evaporative dysfunctional tear syndrome in postmenopausal women: A case-control study. Br J Ophthalmol 98: 371-376, 2014.

20. Klocek MS, Sassani JW, McLaughlin PJ and Zagon IS: Naltrexone and insulin are independently effective but not additive in accelerating corneal epithelial healing in type I diabetic rats. Exp Eye Res 89: 686-692, 2009.

21. Zagon IS, Sassani JW and McLaughlin PJ: Insulin treatment ameliorates impaired corneal reepithelialization in diabetic rats. Diabetes 55: 1141-1147, 2006.

22. Chandramouli C, Reichelt ME, Curl CL, Varma U, Bienvenu LA, Koutsifeli P, Raaijmakers AJA, De Blasio MJ, Qin CX, Jenkins AJ, et al: Diastoli dysfunction is more apparent in STZ-induced diabetic female mice, despite less pronounced hyperglycemia. Sci Rep 8: 2346, 2018.

23. McLaughlin PJ, Sassani JW, Titunick MB and Zagon IS: Efficacy and safety of a novel naltrexone treatment for dry eye in type 1 diabetes. BMC Ophthalmol 19: 35, 2019.

This work is licensed under a Creative Commons Attribution-NonCommercial-NoDerivatives 4.0 International (CC BY-NC-ND 4.0) License. 